\title{
Stationary Patterns in a Two-Protein Reaction-Diffusion System
}

\author{
Philipp Glock, Beatrice Ramm, ${ }^{\circledR}$ Tamara Heermann, ${ }^{\circledR}$ Simon Kretschmer, Jakob Schweizer, \\ Jonas Mücksch, ${ }^{\oplus}$ Gökberk Alagöz, and Petra Schwille*(ఠ)
}

Cellular and Molecular Biophysics, Max-Planck-Institut für Biochemie, Martinsried 82152, Germany

Supporting Information

ABSTRACT: Patterns formed by reaction-diffusion mechanisms are crucial for the development or sustenance of most organisms in nature. Patterns include dynamic waves, but are more often found as static distributions, such as animal skin patterns. Yet, a simplistic biological model system to reproduce and quantitatively investigate static reactiondiffusion patterns has been missing so far. Here, we demonstrate that the Escherichia coli Min system, known for its oscillatory behavior between the cell poles, is under certain conditions capable of transitioning to quasi-stationary protein distributions on membranes closely resembling Turing patterns. We systematically titrated both proteins, MinD and
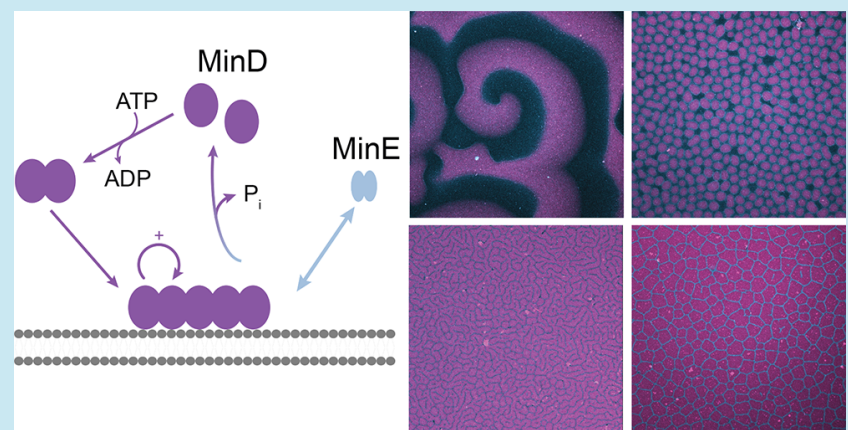

MinE, and found that removing all purification tags and linkers from the N-terminus of MinE was critical for static patterns to occur. At small bulk heights, dynamic patterns dominate, such as in rod-shaped microcompartments. We see implications of this work for studying pattern formation in general, but also for creating artificial gradients as downstream cues in synthetic biology applications.

KEYWORDS: pattern formation, self-organization, in vitro reconstitution, min proteins, reaction-diffusion system, stationary pattern

$\mathrm{P}$ attern formation, the emergence of patterns in initially spatially homogeneous systems, is at the basis of biological systems. It often relies on reaction-diffusion mechanisms, as in the patterning of animal skin ${ }^{1}$ and on multiple occasions in the development of animals. ${ }^{2,3}$ Hence, understanding reactiondiffusion mechanisms in detail is important in the context of cell and developmental biology, but it is mandatory for any fundamental approach toward the emergence of biological systems and for the bottom-up construction of artificial cells.

A prominent candidate for a simple model system to probe reaction-diffusion mechanisms is the Min system of Escherichia coli. The Min proteins perform pole-to-pole oscillations to determine the middle of the cell and future division site. This dynamic behavior results from reaction and diffusion of only two proteins-MinD and MinE-in the presence of and catalyzed by a lipid membrane. Due to its relevance for the intracellular organization of bacteria the system has been studied in depth in vivo. ${ }^{4-6}$ Its apparent simplicity also facilitated the development of multiple mathematical models ${ }^{7,8}$ as well as the reconstitution of the protein dynamics in vitro. The combined efforts resulted in a widely accepted hypothesis for the basic mechanism (Figure 1a): MinD is an ATPase that dimerizes in its ATP-bound state. This renders the membrane affinity of MinD with its weak membrane targeting sequence (MTS) sufficiently high to attach to the membrane, turning the MinD monomer-dimer transition into a membrane switch. ${ }^{10}$ The second protein, MinE, exhibits a latent cytosolic conformation and an active conformation in the presence of membrane-bound MinD, which in turn stimulates the ATPase activity of MinD. MinE thus features its own, MinD-dependent switch. In the active conformation, a small MTS is formed that is hidden in the latent conformation. MinD shows a strong local positive feedback during membrane binding, recruiting further MinD, while MinE negatively regulates MinD membrane binding. Together, this network of interlinked switches promotes stable pattern formation in a large range of concentrations. $^{11}$

The in vitro reconstitution of this system has been used to investigate the dependence of the formed patterns on several parameters, such as membrane charge and buffer composition, ${ }^{12}$ geometry, ${ }^{13-15}$ and the molecular features of Min proteins. ${ }^{11,16,17}$ In all of these investigations, Min proteins were mostly found to self-organize into traveling concentration waves on the surface. ${ }^{9,12}$ While smooth, directionally moving wavefronts can be primarily observed under steady state conditions on homogeneous membranes, additional dynamic patterns have been observed in a flow cell setup. ${ }^{16,18}$ However, despite a decade of reconstitution efforts, stationary patterns with finite wavelength or "Turing" patterns ${ }^{19,20}$ have not been reported for the Min system. As it is known that in reactiondiffusion systems, even relatively minor changes in system parameters can sometimes cause dramatic, qualitative changes in the observed patterns, it was our intention to explore

Received: October 2, 2018

Published: December 20, 2018 


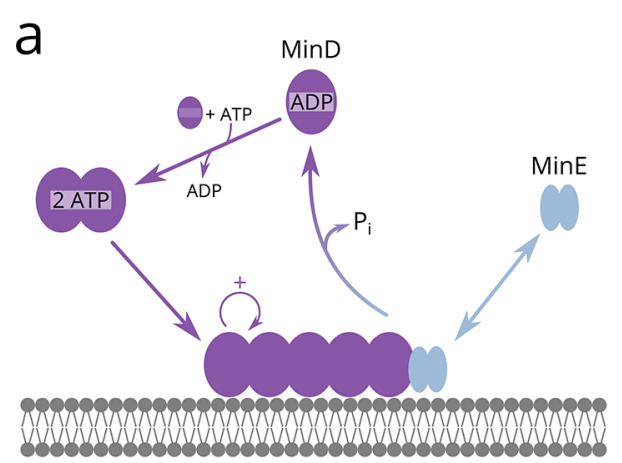

b
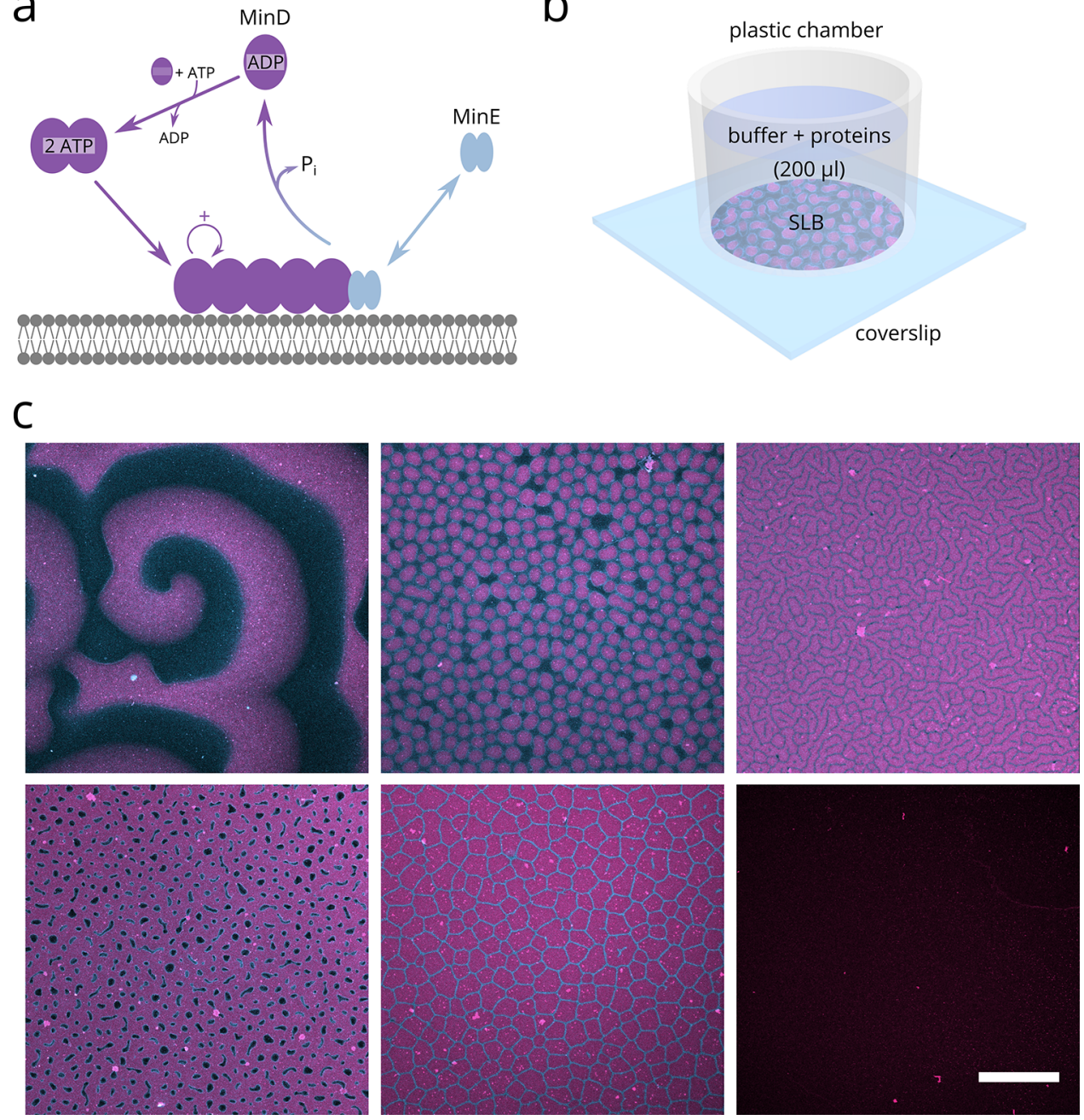

Figure 1. (a) Simplified schematic of Min protein self-organization. MinD (purple) is cytosolic in its ADP-bound state, but attaches to the membrane upon ATP-dependent dimerization. Membrane-bound MinD locally enhances self-recruitment, but also recruits MinE, which activates ATP-hydrolysis and thereby detaches MinD. (b) Experimental setup used: SLB is formed at the bottom of a round plastic chamber glued to a cleaned glass coverslip. Min proteins, ATP, and buffer solution together constitute the bulk volume of $200 \mu \mathrm{L}$. (c) Freeing the N-terminus of MinE leads to the formation of stationary patterns in reconstitution assays: Representative images for each pattern are shown. Top left: Traveling waves, observed at $0.5 \mu \mathrm{M}$ MinD, $1.2 \mu \mathrm{M}$ MinE-His. Top middle: Spots, here at $1.3 \mu \mathrm{M}$ MinD, $4 \mu \mathrm{M}$ MinE-His. Top right: Labyrinth (1.25 $\mu \mathrm{M}$ MinD, $2.5 \mu \mathrm{M}$ MinE-His). Bottom left: Inverse spots (0.5 $\mu \mathrm{M} \mathrm{MinD,} 0.125 \mu \mathrm{M}$ MinE-His). Bottom middle: MinE-mesh (1.5 $\mu \mathrm{M} \mathrm{MinD,} 3 \mu \mathrm{M}$ MinEHis). Bottom right: no pattern (0.2 $\mu \mathrm{M}$ MinD, $1 \mu \mathrm{M}$ MinE-His). (Scale bar: $50 \mu \mathrm{m}$, MinD: magenta, MinE-His: cyan; proteins were fluorescently labeled by doping MinD with 30\% Alexa647-KCK-MinD and MinE-His with 30\% Atto488-KCK-MinE-His, membrane prepared from 2:1 DOPC:DOPG).

whether such a transition from dynamic to static Min patterns could be evoked simply by titration.

Because most protein constructs used for in vitro applications carry additional modifications due to the need for purification, special attention was paid to the influence of such tags, particularly for MinE, which is the smaller protein. Previously, placement of the His-tag at the $\mathrm{N}$ - and C-terminus of MinE showed similar results with respect to pattern formation, although this was examined only at one specific experimental condition. ${ }^{9}$ We therefore revisited our standard MinE construct, which was cloned with an N-terminal His-tag and linker, and systematically investigated pattern formation while titrating the concentrations of MinD and MinE. Strikingly, moving this $\mathrm{N}$-terminal attachment to the $\mathrm{C}$ terminus, or cleaving it off to obtain the wild type protein had an astonishing effect on the observed dynamics in our in vitro assay, and opens up a big spectrum of intriguing patterns that closely resemble Turing patterns. ${ }^{19,20}$ Thus, while the in vitro Min system was believed to be well understood, this new phenomenon of static patterns in such an easily accessible biological reaction-diffusion system offers many exciting perspectives for both experimentalists and theoreticians.

\section{RESULTS AND DISCUSSION}

Reconstituting MinD and MinE-His on a DOPC:DOPG (2:1) supported lipid bilayer in the presence of ATP resulted in a diverse set of patterns (Figure 1c). Traveling surface waves and spirals with the same characteristics as described for His-MinE could be obtained under some conditions. We classified the other, newly observed patterns as spots, labyrinths, inverse spots, and (MinE-) mesh, respectively. At low MinD concentrations, there are regimes where no patterns emerge and the membrane is mostly free of $\mathrm{MinD}$ and MinE (Figure 1c, last panel). Intriguingly, all patterns except the already 


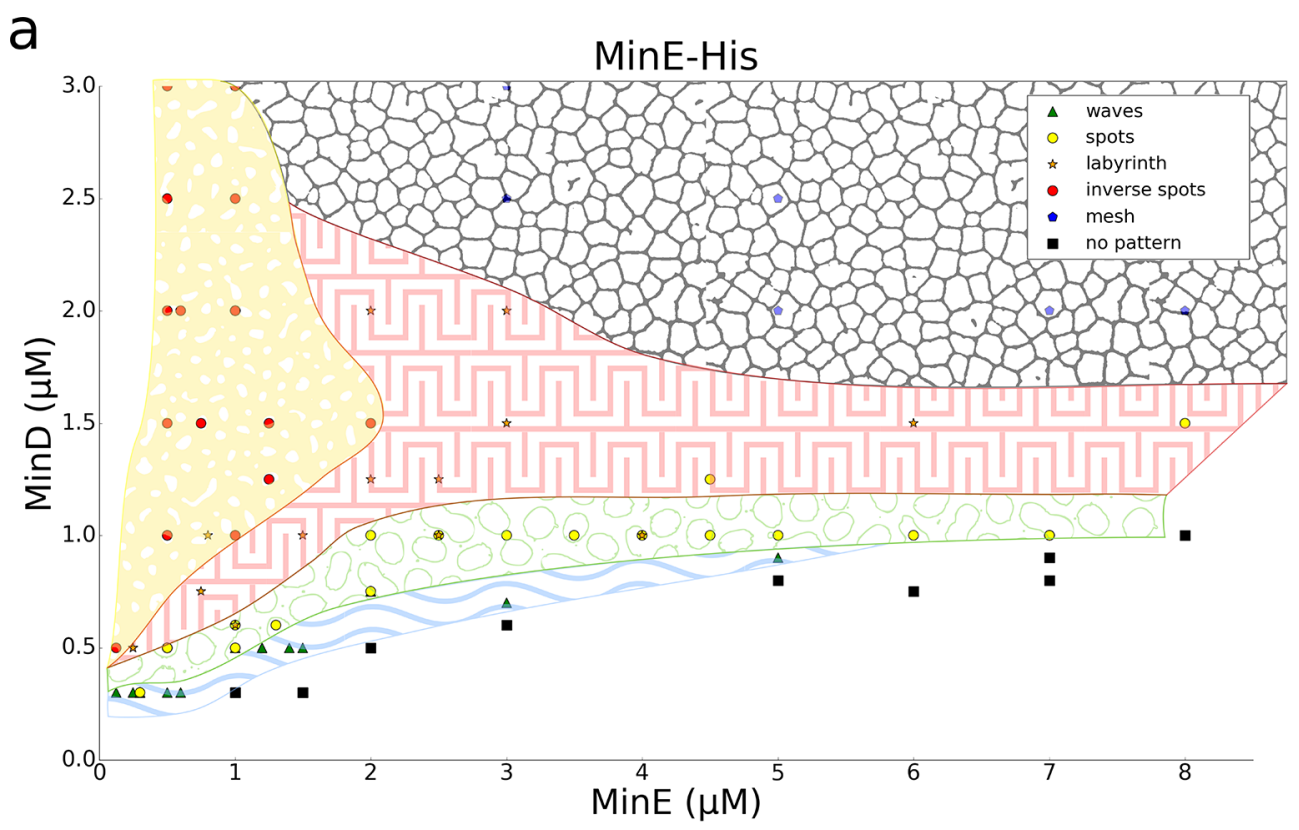

b

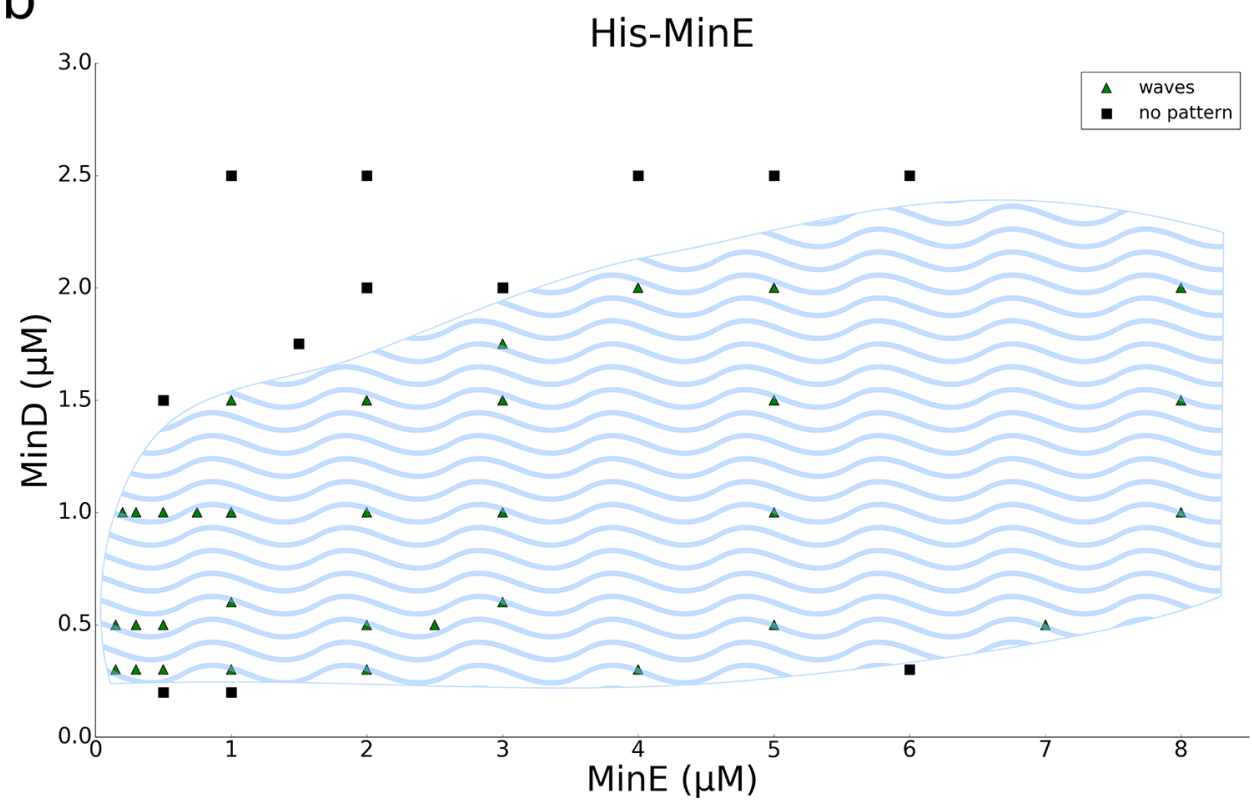

Figure 2. Pattern formation with MinE-His (a) differs considerably from patterns using the previously described His-MinE (b) construct. Varying amounts of MinD (doped with 30\% Alexa647-KCK-MinD) and either MinE-His or His-MinE were reconstituted on 2:1 (DOPC:DOPG) SLBs. Pattern formation was assessed in well-mixed chambers after $1 \mathrm{~h}$ incubation with the proteins and $2.5 \mathrm{mM}$ ATP. The overlaid graphics represent schematic drawings to ease perception of the overall difference between the constructs. Observed patterns are depicted by the symbols described in the legends (top right). Data points depict single observations in separately prepared assay chambers.

described traveling waves are static, and have a strong resemblance to Turing patterns. ${ }^{19,20}$ Importantly, we solely state a qualitative similarity, without drawing conclusions about the mechanism or underlying instability. Gradual small changes can be seen in the patterns (Movie S1), which recover rapidly when photobleached (Movie S2). This shows that, while the overall protein distribution in the large scale pattern is mostly static, there is constant reaction and diffusion occurring, where proteins remain mobile and frequently bind and unbind the membrane. Looking at $\mathrm{MinD}$ and $\mathrm{MinE}$ separately reveals a differential distribution in the static patterns, often with MinE occupying the rim of MinD zones, reminiscent of the in vivo MinE-ring formation and patterns reported by the Mizuuchi lab. ${ }^{18}$ For a separate representation of the membrane distribution of the two proteins in Figure 1c, please refer to Figure S1.

To systematically map out the occurrence of the various patterns as a function of Min protein concentrations, we titrated MinD and MinE-His and summarized the results in a phase diagram (Figure 2a). The striking difference between the two MinE constructs becomes apparent when this plot is compared to a similar diagram compiled from our formerly used protein construct, His-MinE (Figure 2b). Whereas HisMinE, as previously described, self-organizes into traveling 
surface waves in a broad range of concentrations on glass supported lipid bilayers, and does not systematically show additional patterns, MinE-His forms waves only in a narrow range of MinD and MinE concentrations. The newly described patterns occupy most of the area in the phase diagram. The schematic regions depicted on top of the plotted values are simplified representations, where the sharp boundaries between types of patterns are drawn to guide the eye and do not reflect the situation in our assays. Rather, two phenomena should be highlighted specifically: First, the assays show multistability, a phenomenon already reported for axis selection and the number of nodes in confined oscillating Min reactions. ${ }^{21}$ Thus, we commonly observe two adjacent patterns within the same chamber, either separated spatially within the chamber (Figure S3), or temporally with one pattern following minutes to hours after another. This timedependent pattern switch can even happen multiple times within the same, well-mixed assay. Second, while there are strong differences between the individual pattern types (spots, labyrinth, inverse spots, mesh), the transitions between the static patterns show gradual changes, and intermediate patterns are often observed (see also Figure S4). The phase diagram will shift by varying parameters such as membrane composition, buffer ingredients, or support of the lipid bilayer. However, the overall shape of the pattern space is expected to stay the same. We did some experiments using E. coli polar lipid extract to form the membrane, since this mixture has been used in the original publication. Similar to our results with the DOPC:DOPG (2:1) mixture, traveling waves forming with MinE-His occurred at lower MinD/MinE compared to the conditions giving rise to stationary patterns (two conditions found in Figure S5).

To exclude the possibility that static patterns are caused by the C-terminal His-tag, we also generated MinE as it is found in the cell, by expressing it as a His-SUMO-MinE fusion and cleaving it to retrieve the native protein via a selective SUMO protease. The wild type protein produces the same spectrum of patterns as MinE-His, confirming that His-MinE shows a markedly reduced diversity of obtainable patterns relative to wild type MinE and MinE-His (Figure 3). It is important to note that the scale of the patterns varies slightly between assays, even when using the same protein concentrations.

Given the stark difference in pattern diversity between the two MinE constructs, we wondered what kind of dynamic behavior the two proteins show in vivo compared to wildtype MinE. We expressed MinD tagged with superfolder GFP, and either His-MinE, MinE-His, or MinE wt under control of a lac promoter on a low-copy plasmid in $\triangle \operatorname{minDE}$ cells. Analysis of the time-lapse data acquired with these constructs shows that all three constructs support MinD pole-to-pole oscillations in vivo. However, the oscillation periods observed with His-MinE were significantly longer and their spread vastly bigger than measured with wild type MinE or MinE-His (Figure $3 b$, see also Movie S3). On average, cells were also more elongated with His-MinE compared to MinE wt, hinting at a cell division phenotype as a consequence of the altered Min system.

Since the His-tag and linker in His-MinE are directly attached to the MTS, we suspected that a difference in membrane binding might be responsible for the reported changes. Therefore, we performed measurements of MinE binding to a membrane on a quartz crystal microbalance with dissipation monitoring (QCM-D). The data obtained indicate very similar membrane binding by both His-MinE and MinE-

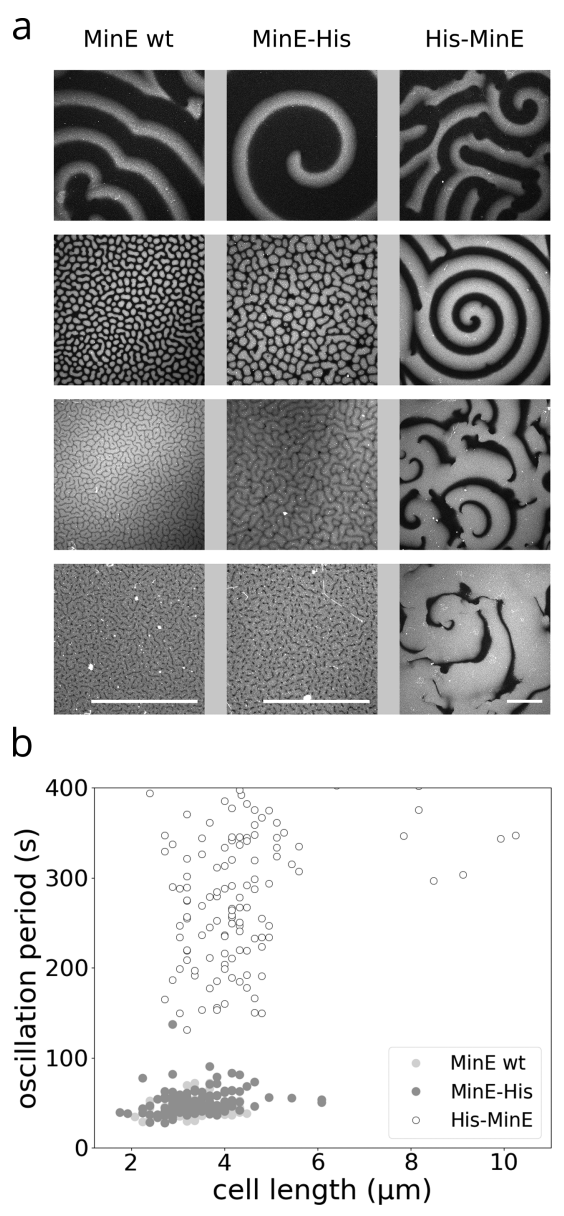

Figure 3. MinE-His and MinE-wt exhibit the same pattern diversity, whereas His-MinE is characterized by impaired pattern diversity in vitro and slower pole-to-pole oscillation in vivo. (a) Comparison of different $\mathrm{MinD}$ and MinE concentrations for all three MinE constructs. Top: $0.5 \mu \mathrm{M}$ MinD, $1.3 \mu \mathrm{M}$ MinE. Second row: $1 \mu \mathrm{M}$ MinD, $3 \mu \mathrm{M}$ MinE. Third row: $1.5 \mu \mathrm{M}$ MinD, $1 \mu \mathrm{M}$ MinE. Bottom row: $1.5 \mu \mathrm{M}$ MinD, $0.5 \mu \mathrm{M}$ MinE. (Scale bars: $50 \mu \mathrm{m}$. All images except left and middle panel in the last row were recorded at the same scale. MinD doped with 30\% Alexa647-KCK-MinD in each case.) (b) In vivo oscillation periods at room temperature relative to cell length induced by the different MinE constructs and superfolder GFP-MinD when expressed in $\Delta(\mathrm{minDE})$ background under IPTG induction. Number of cells measured: MinE wt $(n=104)$, MinE-His $(n=143)$, and His-MinE $(n=189)$. The full plot containing all outliers can be found as Figure S11, and single plots of MinE-His and MinE-wt in Figures S12 and S13.

His as well as untagged MinE (Figure 4a). We therefore conclude that the changes in pattern diversity in vitro and oscillation period in vivo are not due to differential membrane binding of the MinE constructs.

To test whether the linker placement affects the rate of ATP hydrolysis by stimulated MinD, we performed ATPase measurements. And indeed, an NADH-coupled measurement of the ATPase rates revealed that the two MinE constructs lead to a different stimulation of MinD ATPase activity. While the ATPase rate in the presence of His-MinE was measured at roughly $46 \mathrm{nmol}$ ATP per minute and $\mathrm{mg}(\mathrm{MinD})$, it was only about two-thirds as high (28.8 nmol ATP per minute and $\mathrm{mg}$ $(\mathrm{MinD}))$ in the presence of MinE-His and MinE wt. This shows that, in comparison to MinE wt, His-MinE is not impaired but hyper-active, which might be the cause for it to 
a
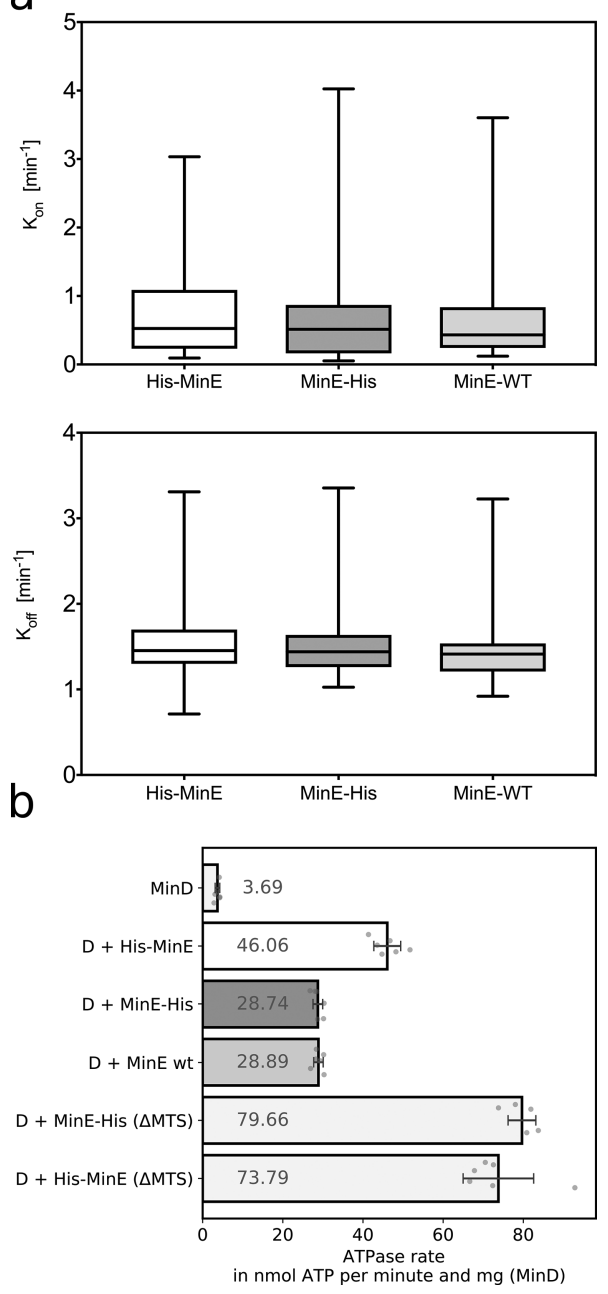

Figure 4. All MinE constructs with MTS bind the membrane with similar affinity, but MinE wt and MinE-His show reduced MinD ATPase stimulation compared to His-MinE. (a) QCM-D measurements of His-MinE, MinE wt, and MinE-His binding to membranes show no apparent difference in membrane binding and unbinding between the constructs. (b) ATPase rates of MinD plus different MinE constructs was measured in an NADH-coupled assay. Data are combined from two independent assays of triplicates for each condition. Error bars represent standard deviation; data points are overlaid.

support dynamic rather than stationary patterns (Figure $4 b$ ). Furthermore, we tested versions of MinE without the MTS, with the His-tag on either end of MinE, and found no significant difference between ATPase rate stimulation by those constructs at the tested concentrations. This further supports the notion that the His-tag does not impede MinE membrane binding.

Recent studies have generated new insights into the conformational dynamics of MinE by employing CD spectroscopy and hydrogen-deuterium exchange coupled to mass spectrometry. $^{22,23}$ The authors suggest that there is no equilibrium between MinE's $6 \beta$-stranded and $4 \beta$-stranded version. Instead, within the $6 \beta$-stranded form, MinE transiently releases its MTS along with a loop segment connecting the MTS to the $\beta$-sheet. In this state, the switch from the $6 \beta$ stranded latent state to the $4 \beta$-stranded reactive state is subsequently triggered if MinE encounters, or "senses", 6 a membrane-bound MinD dimer. In a recent publication, MinE's conformational switch was looked at from a dual theoryexperiment perspective. ${ }^{11}$ By using the $\operatorname{MinE}(\mathrm{I} 24 \mathrm{~N})$ mutant which is permanently locked in the $4 \beta$-conformation, the impact of MinE conformational switching on Min protein pattern formation was investigated. If addition of the $\mathrm{N}$ terminal tag and linker would severely impact switching, similarly compromised pattern formation as with I24N would be expected for His-MinE. Instead, we observed a different phenotype where traveling waves are the only observed mode. We speculate that the higher ATPase rate and different phase diagram of MinE-His in comparison with His-MinE could be attributed to a slower return of the His-linker-MinE from the (reactive) $4 \beta$ to the (latent) $6 \beta$-conformation. This would render MinE hyperactive and lead to a higher ATP turnover.

As a further confirmation that the difference between the MinE constructs is not due to membrane binding, MTSdeficient (missing amino acids 2-12) versions of His-MinE and MinE-His show slightly different self-organization characteristics in our in vitro assay, while the ATPase rate of both constructs remains the same. In particular, while both the $\Delta$ MTS MinE-His and $\Delta$ MTS His-MinE constructs displayed a reduced wavelength compared to the WT under wave conditions, we also noticed a difference in the patterns' spatial scale between the $\mathrm{N}$ - and C-terminally tagged $\triangle \mathrm{MTS}$ constructs at the tested concentrations (Figure S5).

Having noticed all those discrepancies between the differentially tagged versions of MinE (see also Figure S6), we wondered whether important features of in vitro Min patterns established with His-MinE could also be observed with MinEHis and, conversely, if experiments with MinE-His could provide additional insights. Therefore, we thoroughly revisited several key, past experiments.

First of all, the overall shape of the MinD- and MinEdensities along a traveling wave profile remain unchanged by the new construct (Figure S7), consistent with results obtained by the Mizuuchi lab, ${ }^{12}$ who use MinE-His for their experiments. MinE accumulates over the course of a wave and reaches maximal density where MinD density is already decaying. Rapid rebinding of MinE in the traveling wave has so far only been shown with the N-terminally tagged MinE, and should possibly be confirmed with C-terminally tagged MinE by future experiments. ${ }^{16,24}$ However, as highlighted by our phase diagrams (Figure 2), both His-MinE and MinE-His form Min patterns in a similar, broad range of MinE concentrations. $^{11}$

The reconstitution of Min proteins in PDMS microstructures was the first assay to mimic the rod shape of bacterial cells with simultaneous reaction confinement. While the oscillations differ from in vivo oscillations in scale, due to a larger wavelength and reaction volume, they nevertheless reproduce key aspects of in vivo behavior. ${ }^{25}$ Briefly, an SLB is formed on a micropatterned PDMS support. MinD, MinE, and ATP are added into the large bulk volume, and selforganization is first observed everywhere on the membrane without lowering the buffer level. Once regular waves are running on top of the wells, the buffer is lowered, so that individual microcavities become isolated and the proteins and ATP confined within. His-MinE oscillates under these conditions with a very similar time-averaged protein concentration profile as observed in vivo. Performing the same assay with MinE-His also resulted in oscillating microcompartments when choosing $\mathrm{MinD}$ and MinE concentrations that lead to traveling waves in the bulk assay (Figure 
5a). Intriguingly, MinE-His also supports pole-to-pole oscillations and other dynamic patterns when the buffer is

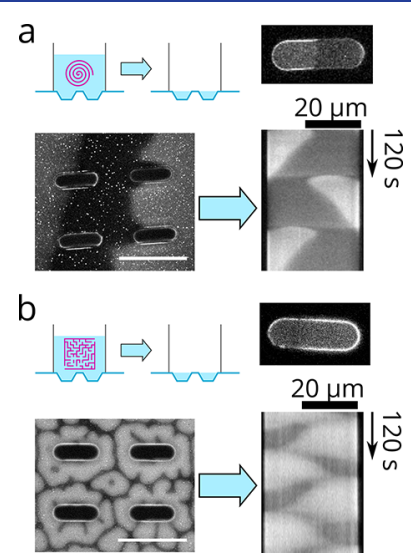

Figure 5. Reaction confinement leads to dynamic oscillations both under wave (a) and static conditions (b). Microchamber assays were performed, and pattern formation was observed before lowering the buffer. Irrespective of the observed pattern before buffer-removal, oscillations were observed in isolated microcompartments. (Concentrations: top $0.75 \mu \mathrm{M}$ MinD, $2 \mu \mathrm{M}$ MinE-His, bottom: $1.3 \mu \mathrm{M} \mathrm{MinD}$, $4 \mu \mathrm{M}$ MinE-His, $20 \%$ Alexa647-KCK-MinD in each, scale bars in overview images: $50 \mu \mathrm{m}$ ).

lowered at conditions that initially form static patterns, such as labyrinths or spots (Figure $5 b$ ). Thus, there is only a minor difference in behavior between His-MinE and MinE-His under conditions where the bulk volume is limited. This is further supported by measurements in a flow cell, where almost exclusively dynamic patterns with MinE-His are observed, presumably due to the much lower bulk volume. ${ }^{12,16,18}$

In a recent study, we showed that the MinDE system can act as a generic cue to position other membrane-bound or membrane-associated factors. ${ }^{26}$ Probing the same effect with MinE-His confirmed the results obtained with His-MinE: The Min system can position model peripheral and lipid-anchored membrane proteins (Figure 6, Movies S5 and S6). The extent of regulation is similar where $\mathrm{MinD}$ and MinE-His form traveling waves (Figure S8). Additionally, the newly observed static patterns strongly exclude other proteins from areas where the membrane-bound MinD levels are high. Importantly, since the distributions of MinD and MinE are distinct in these static patterns, we can now clearly state that membrane-bound MinD alone is responsible for the observed positioning effect. The static patterns can therefore be used as a novel cue to position other membrane-associated proteins in vitro.

On top of this, the static patterns themselves offer intriguing possibilities for all kinds of in vitro investigations. Since the main difference in diffusion of the Min components depends on membrane-binding upon dimerization, the absolute size and bulk diffusion of either component are not crucially important. For example, fluorescent proteins have been added to both MinD and MinE in vivo without disturbing Min oscillations. ${ }^{4,27}$ Thus, attaching for instance functional secondary proteins to a fraction of $\mathrm{MinDs}$ is not likely to impact pattern formation to a relevant degree. This is further evidenced by many of our previous experiments where 20 to $30 \%$ of MinDs were tagged with the fluorescent proteins eGFP or mRuby3..$^{11,17,28}$ One could therefore imagine assays where the Min system merely positions factors in a self-organized manner, which is especially compelling in a case where spots

\section{a}

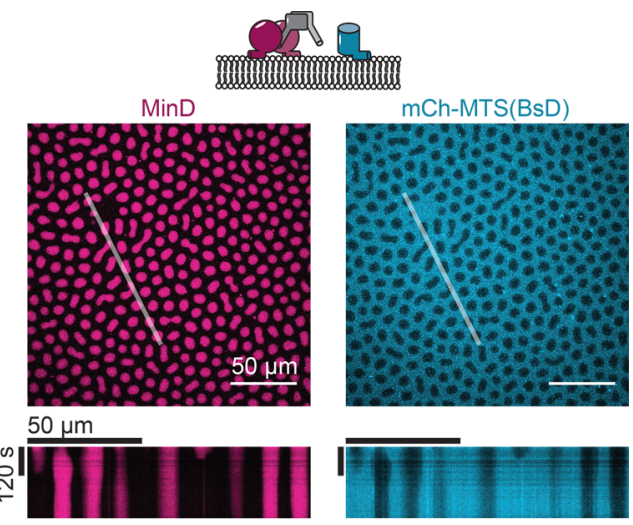

b

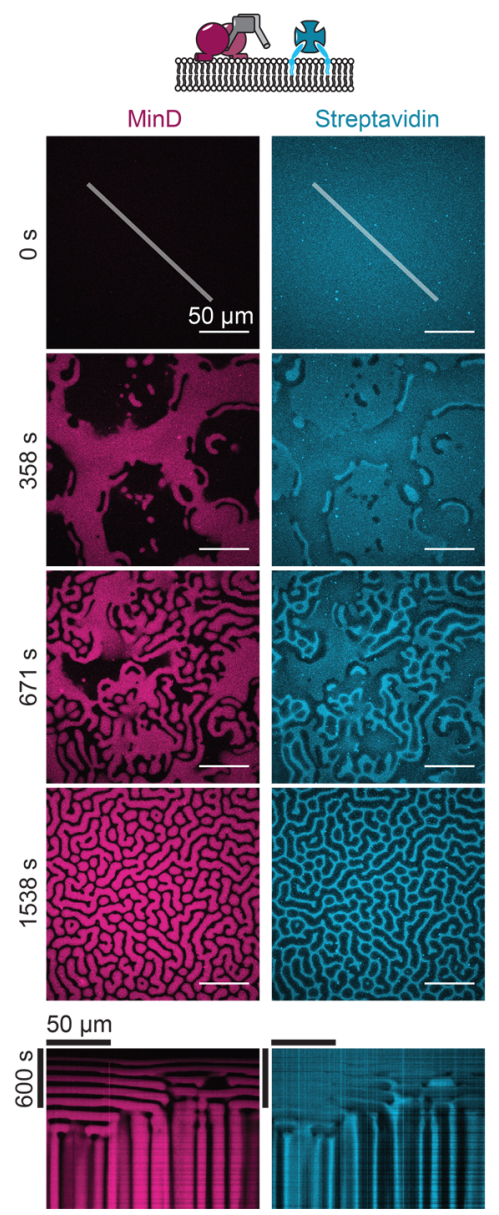

Figure 6. MinD and MinE-His are able to spatiotemporally regulate model peripheral and lipid-anchored proteins. (a) MinDE spot patterns formed with MinE-His exclude the model peripheral membrane protein mCh-MTS(BsD) (0.75 $\mu \mathrm{M} \mathrm{MinD} \mathrm{(30 \%} \mathrm{EGFP-}$ MinD). Two $\mu \mathrm{M}$ MinE-His, $1 \mu \mathrm{M}$ mCh-MTS(BsD)). Kymographs of the respective line selection. (b) MinDE static patterns formed in the presence of MinE-His are spatiotemporally regulating lipid-anchored streptavidin, forming a static inverse streptavidin pattern. Time-series of the establishment of MinDE labyrinth pattern and inverse streptavidin pattern (1 $\mu \mathrm{M} \mathrm{MinD} \mathrm{(30 \%} \mathrm{EGFP-MinD);} 2 \mu \mathrm{M}$ MinE-His, Alexa647-Streptavidin, $69 \mathrm{~mol} \%$ DOPC/30 mol \% DOPG/1 mol \% Biotinyl-CAP-PE). Kymograph along the line selection shown at time $=0 \mathrm{~s}$.

are rendered sparse by adding more MinE-His (Figure S9). These new stationary patterns could be used for example to anchor polymerizing or depolymerizing factors for cytoskeletal proteins like actin or tubulin onto model membranes to create 
new active systems. Another interesting experiment would be to tether any component essential for protein production to the MinD foci and thereby locally produce proteins via cellfree protein production.

Early experiments on mica as a support showed static patterns with His-MinE under certain conditions. These were not further investigated, however. ${ }^{9}$ It is puzzling also to us that the spectrum of intriguing patterns observed with MinE-His has not been reported before, but we have to assume that researchers using similar (large available bulk volume) setups previously tested MinE-His only under conditions of (effectively) low MinD concentration, where it forms traveling waves as observed with His-MinE. In the original paper's supplement, MinE-His is mentioned in that exact context, and no difference to His-MinE was found. ${ }^{9}$ Similarly, Vecchiarelli et al. find that MinE-His forms traveling waves in their flow-cell setup, likely because the bulk height or the total protein amounts are more limited. ${ }^{12}$ This once again highlights how easily discoveries are overlooked and how important it is to test reconstituted systems under multiple conditions. This is particularly important in the case of self-organizing reactiondiffusion networks that are inherently sensitive to changes in parameter values due to the underlying nonlinearity of the molecular interactions. Overall, as also indicated by Figure 3, we are confident that under traveling wave conditions, MinEHis, His-MinE, and MinE-wt show similar characteristics, which validates our past results for the dynamic Min system.

The reported static patterns are unlikely to play a major role for the Min system in live E. coli. However, there are many Gram-positive bacteria, such as B. subtilis, that contain a static Min system ${ }^{29,30}$ that in the light of our results might also form their patterns through a highly dynamic reaction-diffusion mechanism tuned to yield a quasi-static protein distribution. Additionally, static Min patterns show multiple aspects of relevance:

First, their existence is proof of the big versatility of biological reaction-diffusion systems. On the basis of relatively simple physical principles and very few components, they can regulate complex tasks efficiently, while forming entirely different patterns in another context. This, in turn, highlights how important not just the concentration of a protein can become, but also the total available bulk volume, and thus particle number within an enclosure. Positive feedback in MinD recruitment amplifies this effect.

Second, the emergence of static patterns significantly enlarges the functionality toolbox provided by the Min system, making it more attractive for research on reaction-diffusion mechanisms and to use it for designing spatiotemporal cues in bottom-up synthetic biology. Requiring only two proteins, ATP and a membrane makes the system easily accessible, and naturally limits the complexity of mathematical models. Experimentalists interested in pattern formation have so far mostly turned to chemical reactions, like the BelousovZhabotinsky or the chloride-iodide-malonic-acid (CIMA) reaction. Both involve too many components and intermediates to hope for comprehensive modeling or simulating full complexity. In contrast the Min system is truly biological and therefore biocompatible, the proteins can be engineered in countless ways, and the reaction runs stably for many hours, even days without buffer exchanges. Intentional disturbances (example in Movie S4) can be introduced via a recently published photoswitch. ${ }^{28}$ With this in mind, we have recently increased our efforts to make this versatile system available to other researchers by sharing all plasmids and detailed protocols. $^{31}$

\section{METHODS}

Plasmid Design and Cloning. pET28a-MinE-His, pET28a-MinE-His ( $\Delta$ MTS), pET28a-MinE-KCK-His and pET28a-His-KCK-MinD were generated via homologous recombination in E. coli. For more details on the cloning procedure, including primers and all in vivo plasmids, please refer to the Supporting Information. A plasmid map for pET28a-His-MinE has recently been published. ${ }^{31}$

Proteins were expressed and purified as published previously. ${ }^{31}$ Expression of His-SUMO-MinE was done similarly, but protein in elution buffer $(50 \mathrm{mM}$ Tris- $\mathrm{HCl} \mathrm{pH}$ $8,300 \mathrm{mM} \mathrm{NaCl}, 250 \mathrm{mM}$ imidazole, $10 \%$ glycerol, $10 \mathrm{mM} 2-$ mercaptoethanol, EDTA-free protease inhibitor) was dialyzed against final storage buffer ( $50 \mathrm{mM}$ HEPES pH 7.25, $150 \mathrm{mM}$ $\mathrm{KCl}, 10 \%$ glycerol, $0.1 \mathrm{mM}$ EDTA) while adding 1:100 parts of SUMO protease (His6-SenP2). Protease and cleaved tag were removed by adding excess Ni-NTA Agarose beads, incubating for $1-2 \mathrm{~h}$ on a rotating shaker at $4{ }^{\circ} \mathrm{C}$ and taking the supernatant.

Protein Concentration Measurements. Protein concentrations were all determined via a linearized, improved Bradford assay, as described here. ${ }^{32}$ Measurements were done in 96-well plates, and triplicates of each measured concentration were taken. A minimum of two different dilutions of the same protein stock were measured. Concentration determination of multiple proteins was done in the same assay where critical for comparison (all the MinE proteins used for ATPase rate comparison in Figure 4b).

Labeling and Determination of Degree of Labeling. Labeling of Min proteins was performed according to the dye manufacturer's instructions. A ratio of 3:1 dye to protein was found sufficient to achieve the desired labeling efficiency. A PD-10 buffer exchange column was used to remove most of the unbound dye, followed by overnight dialysis against excess storage buffer.

Cleaning of Coverslips. Twenty-four $\times 24 \mathrm{~mm} \# 1.5$ coverslips (obtained from Menzel) were piranha cleaned by adding 7 drops of sulfuric acid plus two drops of $50 \%$ hydrogen peroxide to the center of each coverslip. The reaction was incubated on the coverslips for at least $45 \mathrm{~min}$ before thoroughly rinsing with ultrapure water. For experiments showing the positioning of membrane proteins by MinDE the coverslips were plasma-cleaned with oxygen as a process gas instead of piranha-cleaning.

Assay Chamber Assembly. The bottom half and lid of $0.5 \mathrm{~mL}$ reaction tubes were cut off with sharp scissors. UV-glue (Norland optical adhesive \#68) was applied with a pipet tip to the upper rim of the tube. The tube was then glued upside down onto the clean coverslip and cured under a UV lamp $(365 \mathrm{~nm})$ for $10 \mathrm{~min}$.

Lipid Preparation. DOPC and DOPG as well as E. coli polar lipid extract (EPL) were obtained from Avanti and dissolved in chloroform at $25 \mathrm{mg} / \mathrm{mL}$. Lipids were mixed in a $1.5 \mathrm{~mL}$ glass vial and the lipid film was dried on the lower rim of the glass under a slight nitrogen stream. The lipid film was further dried by applying vacuum for at least $1 \mathrm{~h}$. Dried lipid films were rehydrated in Min buffer $(150 \mathrm{mM} \mathrm{KCl}, 25 \mathrm{mM}$ Tris- $\mathrm{HCl} \mathrm{pH} 7.5,5 \mathrm{mM} \mathrm{MgCl}_{2}$ ) at $4 \mathrm{mg} / \mathrm{mL}$ by vortexing, then further processed by freeze-thawing for 8-10 cycles in liquid nitrogen $/ 90{ }^{\circ} \mathrm{C}$ hot water. The obtained unilamellar 
vesicles were extruded through a membrane with $50 \mathrm{~nm}$ pore size for 37 passes. EPL vesicles were prepared as described previously. ${ }^{17}$ Vesicles were then used, stored in the fridge for up to 2 weeks or frozen in liquid nitrogen, in which case they were briefly sonicated before use.

SLB Formation. Vesicles were diluted by adding $130 \mu \mathrm{L}$ of Min buffer to a $20 \mu \mathrm{L}$ aliquot of clear lipids at $4 \mathrm{mg} / \mathrm{mL}$. $75 \mu \mathrm{L}$ of this mixture were added to a chamber situated on a heat block at $37^{\circ} \mathrm{C}$. After $1 \mathrm{~min}, 150 \mu \mathrm{L}$ of Min buffer was added. After $2 \mathrm{~min}$ (total time: $3 \mathrm{~min}$ ), chambers were washed by adding $200 \mu \mathrm{L}$ of prewarmed Min buffer, mixing a few times and removing $200 \mu \mathrm{L}$ into liquid waste. This procedure was repeated until $2 \mathrm{~mL}$ of buffer per chamber had been used up. Washed SLB chambers were transferred from $37{ }^{\circ} \mathrm{C}$ to room temperature. For experiments shown in Figure S5, cover slides were piranha cleaned as above, and SLBs then prepared as described. ${ }^{17}$

PDMS Microstructures. PDMS microstructures were prepared as previously described. ${ }^{15}$ For a more detailed protocol including a wafer blueprint please refer to a more recent publication. $^{31}$

Microscopy. Images were acquired on a commercial Zeiss LSM 780 microscope with Zeiss $40 \times$ W NA 1.2 PlanApochromat objective and $10 \times$ objectives. All live-cell images were taken on a custom-built TIRFM (total internal reflection fluorescence microscopy) setup as described. ${ }^{28}$

Image Manipulations (FIJ). Microscopic images used in the figures and videos presented in the main text and Supporting Information were adjusted for brightness and contrast, and if necessary for presentation merged from two separately or concurrently recorded channels (e.g., MinD and MinE in Figure 1, MinD and membrane binders in Figure 6).

Spatiotemporal Regulation of Peripheral and LipidAnchored Proteins by MinDE. Experiments were performed as described. ${ }^{26}$ In short, SLBs were formed with $70 \% / 30 \%$ DOPC/DOPG and in case of streptavidin with $1 \%$ BiotinylCAP-PE. For experiments with peripheral membrane proteins MinD and MinE-His with $1 \mu \mathrm{M}$ mCh-MTS(BsD) were incubated on SLBs for $1 \mathrm{~h}$ before image acquisition. For lipidanchored streptavidin, streptavidin was bound to BiotinylCAP-PE SLBs, and excess streptavidin was removed by washing with buffer. The self-organization of MinDE was started by addition of ATP and a time-series was acquired.

Acquisition and analysis of QCMD data are described in the Supporting Methods.

ATPase Assay, NADH Coupled. ATPase rate was measured in an $\mathrm{NADH}$-coupled assay, whereby the decrease in $\mathrm{NADH}$ concentration is monitored, and ATP concentration stays constant. To achieve this, phosphoenolpyruvate (PEP) (2 $\mathrm{mM})$ and $\mathrm{NADH}(0.5 \mathrm{mM})$ were added to the proteins (all at $2 \mu \mathrm{M})$ in Min buffer (25 mM Tris- $\mathrm{HCl} \mathrm{pH} \mathrm{7.5,} 150 \mathrm{mM} \mathrm{KCl}$, $5 \mathrm{mM} \mathrm{MgCl}_{2}$ ). Vesicles at $50 \mathrm{~nm}$ diameter (via extrusion) were added at a final concentration of $0.2 \mathrm{mg} / \mathrm{mL}$. Additionally, $4.5 \mu \mathrm{L}$ per well of a commercial enzyme mix containing pyruvate kinase $(600-1000 \mathrm{U} / \mathrm{mL})$ and lactate dehydrogenase $(1000-1400 \mathrm{U} / \mathrm{mL})$ were added to facilitate the reaction of ADP with PEP to pyruvate and ATP, and the reduction of pyruvate using $\mathrm{NADH}$ to L-lactate and $\mathrm{NAD}^{+}$. A 96-well plate format at $150 \mu \mathrm{L}$ assay volume was used to measure all conditions concurrently and in triplicates. Decrease in absorption at $340 \mathrm{~nm}$ was measured to calculate the ATPase activity. NADH decomposition due to reasons other than ATPase activity was measured each time in control wells and subtracted from the apparent ATPase rates. Only the linear parts of the measured values were used to obtain the ATPase rate. Note: ATPase rates reported here should only be interpreted as a relative comparison between MinE constructs. There is a large batch-to-batch variation in MinD's ATPase activity.

Bacterial Transformation and Agar Pads for Microscopy, Imaging. Live-cell imaging was performed with strain HL1 ( $\Delta$ minDE zcf1 17::Tn10 recA::cat. ${ }^{33} \mathrm{HL1}$ was transformed with the plasmids pMLB_sfGFP_MinDMinE, pMLB_sfGFPMinD N-His-linker MinE and $\bar{p}$ MLB_sfGFP-MinD $-\overline{M i n E}$. Cells were inoculated from glycerol stocks and grown overnight in LB medium supplemented with ampicillin and tetracycline for $14-16 \mathrm{~h}$ at $37^{\circ} \mathrm{C}$ and $270 \mathrm{rpm}$. Subsequently cells were diluted 1:200 in $20 \mathrm{~mL}$ fresh M9 medium (1X Gibco M9 minimal Salts (Thermo Fisher Scientific, Waltham, USA), $0.2 \%$ casamino acids (Amresco, Solon, Ohio), 0.4\% glycerol, $2 \mathrm{mM} \mathrm{MgSO}_{4}, 0.1 \mathrm{mM} \mathrm{CaCl} 2$ ) supplemented with ampicillin and grown at $37{ }^{\circ} \mathrm{C}$. When the cells reached an OD600 of $\sim 0.1$, MinD and MinE expression was induced by addition of $50 \mu \mathrm{M}$ IPTG. Cell were grown for another $2-3 \mathrm{~h}$ until they reached an OD600 of $\sim 0.3$. They were then diluted in fresh M9 medium to an OD600 of 0.1 and $1 \mu \mathrm{L}$ of the cell suspension was spotted on agar pads and dried for at least 20 min at room temperature, before the agar pads were flipped onto a glass-bottom dish (ibidi), that was lidded for imaging. Imaging settings and time intervals were optimized for each strain to acquire the maximum amount of oscillations with least bleaching.

Twenty-two by $22 \mathrm{~mm}$ coverslips were cleaned with $99 \%$ ethanol and $\mathrm{ddH}_{2} \mathrm{O}$. For preparation of agarose pads M9 media with $1.5 \%(\mathrm{w} / \mathrm{v})$ UltraPure Low Melting Point Agarose (Life Technologies, Carlsbad, CA) was heated in the microwave and then allowed to cool before addition of the appropriate antibiotics and $50 \mu \mathrm{M}$ IPTG. $800 \mu \mathrm{L}$ of the liquid agarose mixture was pipetted onto a 22 by $22 \mathrm{~mm}$ coverslip positioned on a dime. Another coverslip was immediately placed on top yielding an evenly thick agarose coverslip sandwich. Pads were covered and left to solidify at room temperature for about $60 \mathrm{~min}$. The top coverslip was removed and the pad was cut into 16 evenly sized, squared pads with a razor blade.

Analysis of bacterial oscillations using MicrobeTracker ${ }^{34}$ and MATLAB is described in the Supporting Information.

\section{ASSOCIATED CONTENT}

\section{Supporting Information}

The Supporting Information is available free of charge on the ACS Publications website at DOI: 10.1021/acssynbio.8b00415.

Figures S1-S13; Tables S1 and S2; Supporting Methods (PDF)

Movie S1 (AVI)

Movie S2 (AVI)

Movie S3 (AVI)

Movie S4 (AVI)

Movie S5 (AVI)

Movie S6 (AVI)

\section{AUTHOR INFORMATION}

\section{Corresponding Author}

*E-mail: schwille@biochem.mpg.de. 


\section{ORCID}

Philipp Glock: 0000-0002-0238-2634

Beatrice Ramm: 0000-0002-7402-1942

Tamara Heermann: 0000-0003-1607-0727

Jonas Mücksch: 0000-0002-1469-6956

Petra Schwille: 0000-0002-6106-4847

\section{Author Contributions}

P.G. and P.S. conceived the study, P.G. wrote the initial draft, P.G. performed self-organization assays generating data for phase diagrams and microscopy images and performed ATPase assays, B.R. and P.G. performed experiments regarding spatiotemporal regulation and reaction confinement in rodshaped microcompartments, B.R. performed experiments concerning regulation of other membrane-bound proteins by Min reaction, S.K. performed self-organization assays on E. coli polar lipids, G.A. and B.R. performed in vivo experiments, T.H. performed QCM-D experiments and J.M. and T.H. analyzed QCM-D data, J.S. analyzed in vivo data, and P.G., B.R., T.H., S.K., and P.S. discussed and interpreted the results. All authors revised the manuscript.

\section{Notes}

The authors declare no competing financial interest.

\section{ACKNOWLEDGMENTS}

We would like to thank Core Facility MPIB for help with protein purifications, Katharina Nakel for assistance with cloning, Philipp Blumhardt for assistance with live-cell imaging, Anthony Vecchiarelli for sharing plasmid maps, and Piet de Boer for providing strain HL1 and plasmid pDR122. P.G. acknowledges support from the DFG-funded GRK2062 "Molecular principles of synthetic biology". P.G. and J.M. are part of IMPRS-LS. We acknowledge further financial support from the DFG via the Graduate School of Quantitative Biosciences Munich (QBM) (S.K. and B.R.) and the project A09 of the SFB1032 "Nanoagents for the spatiotemporal control of molecular and cellular reactions" (S.K., T.H., and P.S.).

\section{REFERENCES}

(1) Kondo, S., Iwashita, M., and Yamaguchi, M. (2009) How Animals Get Their Skin Patterns: Fish Pigment Pattern as a Live Turing Wave. Int. J. Dev. Biol. 53 (5-6), 851-856.

(2) Economou, A. D., Ohazama, A., Porntaveetus, T., Sharpe, P. T., Kondo, S., Basson, M. A., Gritli-Linde, A., Cobourne, M. T., and Green, J. B. A. (2012) Periodic Stripe Formation by a Turing Mechanism Operating at Growth Zones in the Mammalian Palate. Nat. Genet. 44 (3), 348-351.

(3) Sheth, R., Marcon, L., Bastida, M. F., Junco, M., Quintana, L., Dahn, R., Kmita, M., Sharpe, J., and Ros, M. A. (2012) Hox Genes Regulate Digit Patterning by Controlling the Wavelength of a TuringType Mechanism. Science 338 (6113), 1476-1480.

(4) Raskin, D. M., and de Boer, P. a. (1999) Rapid Pole-to-Pole Oscillation of a Protein Required for Directing Division to the Middle of Escherichia Coli. Proc. Natl. Acad. Sci. U. S. A. 96 (9), 4971-4976.

(5) Szeto, T. H., Rowland, S. L., Habrukowich, C. L., and King, G. F. (2003) The MinD Membrane Targeting Sequence Is a Transplantable Lipid-Binding Helix. J. Biol. Chem. 278 (41), 40050-40056.

(6) Park, K. T., Wu, W., Battaile, K. P., Lovell, S., Holyoak, T., and Lutkenhaus, J. (2011) The Min Oscillator Uses MinD-Dependent Conformational Changes in MinE to Spatially Regulate Cytokinesis. Cell 146 (3), 396-407.

(7) Halatek, J., and Frey, E. (2012) Highly Canalized MinD Transfer and MinE Sequestration Explain the Origin of Robust MinCDEProtein Dynamics. Cell Rep. 1 (6), 741-752.
(8) Halatek, J., and Frey, E. (2018) Rethinking Pattern Formation in Reaction-diffusion Systems. Nat. Phys. 14 (5), 507-514.

(9) Loose, M., Fischer-Friedrich, E., Ries, J., Kruse, K., and Schwille, P. (2008) Spatial Regulators for Bacterial Cell Division Self-Organize into Surface Waves in Vitro. Science 320 (5877), 789-792.

(10) Lackner, L. L., Raskin, D. M., and De Boer, P. a J. (2003) ATPDependent Interactions between Escherichia Coli Min Proteins and the Phospholipid Membrane in Vitro. J. Bacteriol. 185 (3), 735-749.

(11) Denk, J., Kretschmer, S., Halatek, J., Hartl, C., Schwille, P., and Frey, E. (2018) MinE Conformational Switching Confers Robustness on Self-Organized Min Protein Patterns. Proc. Natl. Acad. Sci. U. S. A. 115 (18), 4553-4558.

(12) Vecchiarelli, A. G., Li, M., Mizuuchi, M., and Mizuuchi, K. (2014) Differential Affinities of MinD and MinE to Anionic Phospholipid Influence Min Patterning Dynamics in Vitro. Mol. Microbiol. 93 (3), 453-463.

(13) Schweizer, J., Loose, M., Bonny, M., Kruse, K., Monch, I., and Schwille, P. (2012) Geometry Sensing by Self-Organized Protein Patterns. Proc. Natl. Acad. Sci. U. S. A. 109 (38), 15283-15288.

(14) Caspi, Y., and Dekker, C. (2016) Mapping out Min Protein Patterns in Fully Confined Fluidic Chambers. eLife 5, 1-27.

(15) Zieske, K., and Schwille, P. (2013) Reconstitution of Pole-toPole Oscillations of Min Proteins in Microengineered Polydimethylsiloxane Compartments. Angew. Chem., Int. Ed. 52 (1), 459-462.

(16) Vecchiarelli, A. G., Li, M., Mizuuchi, M., Hwang, L. C., Seol, Y., Neuman, K. C., and Mizuuchi, K. (2016) Membrane-Bound MinDE Complex Acts as a Toggle Switch That Drives Min Oscillation Coupled to Cytoplasmic Depletion of MinD. Proc. Natl. Acad. Sci. U. S. A. 113 (11), E1479-E1488.

(17) Kretschmer, S., Zieske, K., and Schwille, P. (2017) Large-Scale Modulation of Reconstituted Min Protein Patterns and Gradients by Defined Mutations in MinE's Membrane Targeting Sequence. PLoS One 12, 1-16.

(18) Ivanov, V., and Mizuuchi, K. (2010) Multiple Modes of Interconverting Dynamic Pattern Formation by Bacterial Cell Division Proteins. Proc. Natl. Acad. Sci. U. S. A. 107 (18), 8071-8078.

(19) Turing, A. M. (1952) The Chemical Basis of Morphogenesis. Philos. Trans. R. Soc. B Biol. Sci. 237 (641), 37-72.

(20) Kondo, S., and Miura, T. (2010) Reaction-Diffusion Model as a Framework for Understanding Biological Pattern Formation. Science 329 (5999), 1616-1620.

(21) Wu, F., Halatek, J., Reiter, M., Kingma, E., Frey, E., and Dekker, C. (2016) Multistability and Dynamic Transitions of Intracellular Min Protein Patterns. Mol. Syst. Biol. 12 (6), 873.

(22) Ayed, S. H., Cloutier, A. D., McLeod, L. J., Foo, A. C. Y., Damry, A. M., and Goto, N. K. (2017) Dissecting the Role of Conformational Change and Membrane Binding by the Bacterial Cell Division Regulator MinE in the Stimulation of MinD ATPase Activity. J. Biol. Chem. 292 (50), 20732-20743.

(23) Park, K.-T., Villar, M. T., Artigues, A., and Lutkenhaus, J. (2017) MinE Conformational Dynamics Regulate Membrane Binding, MinD Interaction, and Min Oscillation. Proc. Natl. Acad. Sci. U. S. A. 114 (29), 7497-7504.

(24) Loose, M., Fischer-Friedrich, E., Herold, C., Kruse, K., and Schwille, P. (2011) Min Protein Patterns Emerge from Rapid Rebinding and Membrane Interaction of MinE. Nat. Struct. Mol. Biol. 18 (5), 577-583.

(25) Zieske, K., and Schwille, P. (2014) Reconstitution of SelfOrganizing Protein Gradients as Spatial Cues in Cell-Free Systems. eLife 3, 1-19.

(26) Ramm, B., Glock, P., Mücksch, J., Blumhardt, P., GarcíaSoriano, D. A., Heymann, M., and Schwille, P. (2018) The MinDE System Is a Generic Spatial Cue for Membrane Protein Distribution in Vitro. Nat. Commun. 9 (1), 3942.

(27) Raskin, D. M., and de Boer, P. a. (1997) The MinE Ring: An FtsZ-Independent Cell Structure Required for Selection of the Correct Division Site in E. Coli. Cell 91 (5), 685-694.

(28) Glock, P., Broichhagen, J., Kretschmer, S., Blumhardt, P., Mücksch, J., Trauner, D., and Schwille, P. (2018) Optical Control of a 
Biological Reaction-Diffusion System. Angew. Chem., Int. Ed. 57 (9), 2362-2366.

(29) Edwards, D. H., and Errington, J. (1997) The Bacillus Subtilis DivIVA Protein Targets to the Division Septum and Controls the Site Specificity of Cell Division. Mol. Microbiol. 24 (5), 905-915.

(30) Bramkamp, M., Emmins, R., Weston, L., Donovan, C., Daniel, R. A., and Errington, J. (2008) A Novel Component of the DivisionSite Selection System of Bacillus Subtilis and a New Mode of Action for the Division Inhibitor MinCD. Mol. Microbiol. 70 (6), 1556-1569.

(31) Ramm, B., Glock, P., and Schwille, P. (2018) In Vitro Reconstitution of Self-Organizing Protein Patterns on Supported Lipid Bilayers. J. Visualized Exp. No. 137, e58139.

(32) Ernst, O., and Zor, T. (2010) Linearization of the Bradford Protein Assay. J. Visualized Exp. No. 38, 1-6.

(33) Hale, C. A., Meinhardt, H., and de Boer, P. A. (2001) Dynamic Localization Cycle of the Cell Division Regulator MinE in Escherichia Coli. EMBO J. 20 (7), 1563-1572.

(34) Sliusarenko, O., Heinritz, J., Emonet, T., and Jacobs-Wagner, C. (2011) High-Throughput, Subpixel Precision Analysis of Bacterial Morphogenesis and Intracellular Spatio-Temporal Dynamics. Mol. Microbiol. 80 (3), 612-627. 